\title{
Shock-induced polarization in distilled water
}

\author{
Yu. Skryl, ${ }^{1}$ Anna A. Belak, ${ }^{2}$ and Maija M. Kuklja ${ }^{3, *}$ \\ ${ }^{1}$ Institute of Mathematics and Computer Science, University of Latvia, Rìga, LV-1459, Latvia \\ ${ }^{2}$ Virginia Polytechnic Institute and State University, Blacksburg, Virginia 24060, USA \\ ${ }^{3}$ Division of Materials Research, National Science Foundation, Arlington, Virginia 22230, USA \\ (Received 17 January 2007; revised manuscript received 19 May 2007; published 13 August 2007)
}

\begin{abstract}
A detailed analysis of experimental work on shock induced polarization in water performed by Eichelberger and Hauver in 1961 is presented based on an orientation polarization mechanism of water, which is analytically described here in great detail. First, the notion of co-existence of stress and inertial forces is introduced to explain a shock-induced alignment of water molecules in such a way that hydrogen atoms are always moving in front of oxygen atoms along with the shock wave front progressing across the material. Next, the structure of the shock wave front is considered and, specifically, the calculated width of the front is discussed. Furthermore, the polarization induced by shock is computed based on the stress and inertial contributions into the total energy and specific parameters of the shock wave in water. This allows us to define the polarization charge and to describe the formation of the double charged layer within the shock front or distributed over the entire sample depending on the charge relaxation time, which in turn is determined by the dynamics of water viscosity behind the front. The polarization characteristics of water, or electric field, potential, and bias current, calculated here from the polarization charge density, agree well with experimental data by Eichelberger and Hauver. Finally, it is concluded that the reorientation of water molecules is a dominating mechanism of shock-induced polarization. Specific potential applications of the methodology developed here are discussed.
\end{abstract}

DOI: 10.1103/PhysRevB.76.064107

PACS number(s): 46.15. $-\mathrm{x}, 47.40 . \mathrm{Nm}, 66.10 .-\mathrm{x}$

\section{INTRODUCTION}

Eichelberger and Hauver measured a shock-induced electrical polarization in distilled water and proposed that the shock wave is able to reorient molecules and that the atomic masses play the key role in this process. ${ }^{1}$ In addition, they attracted attention to the fact that in some cases "the direction of the signal" was reversed, thus, for example, thallous nitrate yielded a signal very similar to that obtained with water, but with the opposite polarity. ${ }^{1}$

Harris postulated electromechanical coupling to explain the polarization signal resulting from the shock loading of nonpiezoelectric materials. ${ }^{2}$ Although he also suggested that the sign of the polarization is determined by the anion and cation mass difference and that the predicted polarization voltage vanishes for an equal mass ionic crystal; this fact did not follow from their model. Later, Horie, in an attempt to explain Eichelberger and Hauver's experiments, argued that mass asymmetry can not be advanced as the cause of polarization and proposed a model based upon the idea of a local crystalline state of water molecules in which a given molecule was suspended freely at the center of a tetrahedron. ${ }^{3}$ They assumed that a certain rotational freedom of the molecule is suppressed by the uniaxial shock compression, and, therefore, the net polarization appears in the direction of the shock propagation. Harris and Presles analyzed the water data from literature and postulated the existence of a transition from the network of hydrogen bonded water molecules into the network of unbonded dipoles at a given pressure of the shock wave. ${ }^{4}$ This rearrangement is crucial in allowing for a perfect alignment of dipoles contributing to induced polarization signal in the direction of shock propagation within the shock front rise time of approximately $10^{-12} \mathrm{s.}{ }^{4}$

Although the proposed models for the water polarization use quite a natural idea of a reorientation of water molecules in the shock wave and intuitively assume that the mass difference and inertia should be important in the process of forming the polarization signal, they do not offer a clear explanation of shock induced polarization of water, which would be consistent with observed experimental data. Moreover, those theories are not transferable to other materials. There is a wealth of experimental studies on polarization of shocked liquids ${ }^{5-7}$ and solid polar dielectrics. ${ }^{8-10}$ Most of the time, an interpretation of those experiments is based on phenomenological models by Allison, ${ }^{11}$ Zel'dovich, ${ }^{12}$ and others. $^{13,14}$

In this work, a consistent mechanism for polarization of distilled water is developed based on an idea that the alignment of water molecules is caused by the field of stress and inertial forces induced by the shock wave loading. Originally, the idea of the interplay between stress and inertial forces was introduced for the description of point defect diffusion in a shocked organic molecular crystal $\mathrm{C}_{4} \mathrm{H}_{8} \mathrm{~N}_{8} \mathrm{O}_{8}$ (HMX) ${ }^{15}$ Unlike earlier models, an existence of the reorientation of polar molecules in the shock front and its dependence on the atomic mass difference follow directly from taking into account stress and inertial fields rather than postulating them. The method for calculation of shock-induced polarization, electric potential, and bias current in shocked water is developed. Calculated polarization currents quantitatively and qualitatively agree with experimental data by Eichelberger and Hauver. Finally, this study provides an unambiguous interpretation for the experimentally observed inversion of polarization signal, which was not explained before.

\section{CALCULATION OF POLARIZATION}

The water molecule is polar with constitutive atoms of opposite charge, which have different masses. In accordance 


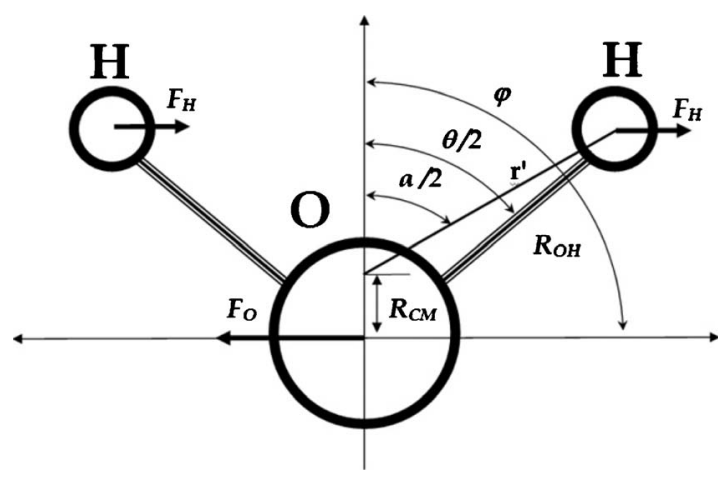

FIG. 1. Free body diagram of the water molecule. Shockinduced net stress and inertial forces exerted on hydrogen and oxygen atoms are shown.

with the proposed ability of polar molecules to reorient themselves in the shock wave ${ }^{1}$ and the notion of coexistence of inertial and stress forces induced by the shock wave, ${ }^{15}$ the water molecules should align along the wave motion and become polarized. Previously, an idea of the importance of an inertial field in materials was introduced to describe diffusion of point defects triggered by the shock wave excitation in the organic molecular crystal, HMX. ${ }^{16}$ Fundamentally, the polarization mechanism of liquid in the field of inertial and stress forces should not differ from the polarization mechanism in an electric field. In order to estimate polarization, it is necessary, therefore, to calculate the work expended to rotate a water molecule in the stress-inertial field.

Let us consider the water molecule in the wave front (Fig. 1). The net forces acting on the hydrogen atom $F_{\mathrm{H}}$ and the oxygen atom $F_{\mathrm{O}}$ are shown by arrows. They point in opposite directions because the inertial force acts mainly on the oxygen atom while the stress force acts on the hydrogen atom due to the mass difference. Defining $R_{\mathrm{MC}}$ as a coordinate of the mass center of the molecule, $R_{\mathrm{OH}}$ as an $\mathrm{O}-\mathrm{H}$ chemical bond length, and $\theta$ as the angle between the two $\mathrm{O}-\mathrm{H}$ bonds, one can express an increase in work $d A$ necessary to rotate the molecule by angle $d \alpha$ due to the forces $F_{\mathrm{O}}$ and $F_{\mathrm{H}}$ as

$$
\begin{aligned}
d A= & F d l=F_{\mathrm{H}} \sin \left(\alpha-\frac{\theta}{2}\right) R_{\mathrm{OH}} d \alpha+F_{\mathrm{H}} \sin \left(\alpha+\frac{\theta}{2}\right) R_{\mathrm{OH}} d \alpha \\
& -F_{\mathrm{O}} \sin (\alpha) R_{\mathrm{MC}} d \alpha,
\end{aligned}
$$

where approximations $r^{\prime} \approx R_{\mathrm{OH}}$ and $\theta^{\prime} \approx \theta$ have been used (see Fig. 1). Equation (1) can be simplified if one introduces new variables $l_{0}=R_{\mathrm{OH}} \cos (\theta / 2)$ and $K=2 m_{\mathrm{H}} / m_{\mathrm{O}}$, were $m_{\mathrm{H}}$ and $m_{\mathrm{O}}$ are atomic masses of hydrogen and oxygen, respectively. Taking into account that $R_{\mathrm{MC}}=K l_{0}$, the increase in work will be written as

$$
d A=\left(2 F_{\mathrm{H}}-K F_{\mathrm{O}}\right) l_{0} \sin (\alpha) d \alpha .
$$

Now, the polarization $P$ can be found once $d A$ is known as well as the concentration of dipoles $N$ with dipole moments $P_{0}$ by using an averaging procedure ${ }^{17}$

$$
P=\tilde{p} N=N \frac{\int_{0}^{\pi} \exp \left(-\frac{A}{k_{B} T}\right) p(\alpha) \sin \alpha d \alpha}{\int_{0}^{\pi} \exp \left(-\frac{A}{k_{B} T}\right) \sin \alpha d \alpha},
$$

where $p(\alpha)=P_{0} \cos (\alpha)$ is the projection of the dipole moment on the direction of the shock wave propagation. Representing the work as $A=-\left(2 F_{\mathrm{H}}-K F_{\mathrm{O}}\right) l_{0} \cos (\alpha)$ after integration of Eq. (2), one finds a well known Langevin form of the polarization expression:

$$
\begin{gathered}
P=-N P_{0}\left\{\operatorname{cth}(z)-\frac{1}{z}\right\}, \\
z=\frac{l_{0}}{k_{B} T}\left(2 F_{\mathrm{H}}-K F_{\mathrm{O}}\right) .
\end{gathered}
$$

In the case of $z \ll 1$, which is always satisfied since $l_{0}$ is on the order of an $\AA, \operatorname{cth}(z)=1 / z+z / 3$ and the formula for polarization is simplified further:

$$
P=\frac{l_{0} P_{0} N}{3 k_{B} T}\left(2 F_{\mathrm{H}}-K F_{\mathrm{O}}\right)=-\frac{l_{0} P_{0} N}{3 k_{B} T} \frac{\partial}{\partial x}\left(2 \varepsilon_{\mathrm{H}}-K \varepsilon_{\mathrm{O}}\right) .
$$

The energies of the hydrogen atom $\varepsilon_{\mathrm{H}}$ and oxygen atom $\varepsilon_{\mathrm{O}}$ are defined as the corresponding differences of their stress and kinetic energies $\left(\varepsilon_{\mathrm{H}}=\varepsilon_{\mathrm{H} s}-\varepsilon_{\mathrm{H} k}\right.$ and $\left.\varepsilon_{\mathrm{O}}=\varepsilon_{\mathrm{O} s}-\varepsilon_{\mathrm{O} k}\right)$ and can be found from the known parameters of the shock wave (see the Appendix) as

$$
\begin{gathered}
\varepsilon_{i s}=\rho_{0} C V_{i}\left\{\frac{\gamma_{0}-2 S}{2 S^{2}}\left[\frac{1}{S} \ln (1-\mu S)+\mu\right]+\frac{\gamma_{0}}{4 S^{2}} \mu^{2}\right\}, \\
\varepsilon_{i k}=\frac{1}{2} m_{i} \nu^{2},
\end{gathered}
$$

where $V_{i}$ and $m_{i}$ are the effective volume and the mass of the atoms under study $(i=\mathrm{H} ; \mathrm{O})$. Equation (7) is obtained by integrating the elastic pressure from Eq. (A1) with $f(\mu)$ defined for water (see the Appendix).

An important conclusion follows from Eq. (7); the shock wave always induces polarization in a sample if it constitutes of polar molecules. Alignment of molecules by viscous forces and by viscoelastic forces were described earlier by Enikeev. ${ }^{18}$ The current study demonstrates that the inclusion of an inertial field is crucial for a complete description of polarization as only the existence of inertial forces reveals a correlation between polarization signal and the mass of charged atoms (molecules). This discovered correlation provides an unambiguous interpretation of the experiments by Eichelberger and Hauver, who noted that thallous nitrate (which has a greater mass at the positively charged location) yields a signal very similar to that obtained with water (in which the positively charged part of the molecule was associated with atoms of low mass), except that the polarity is reversed. ${ }^{1}$ From Eq. (6), the inversion of the polarization signal (i.e., change of the polarization sign in the equation) 
explicitly occurs once the atom with the greater mass at the positively charged location is replaced with a positively charged atom of lower mass.

\section{CALCULATION OF POTENTIAL, ELECTRIC FIELD, AND BIAS CURRENT}

For a detailed quantitative comparison of the suggested polarization mechanism with the experiment, one needs to calculate the electric field, potential, and bias current induced in water by the shock wave. The magnitude of the electric field and potential induced in a sample under shock loading can be calculated from the known polarization $P$. An alternative way is to derive those values from the density of the space polarization charge as a function of relaxation time. We define relaxation time to be the time needed for the induced polarization charge to decay from its maximum to zero. During this time, water molecules that were aligned by the shock front relax back to their equilibrium, losing their ordered dipole arrangement. Intuitively, it appears that this process strongly depends on viscosity, which in turn changes with the shock front progressing across the sample. The viscosity of water increases behind the shock front and therefore the process of charge decay will most likely go slowly. To obtain an analytic formula describing the water viscosity as a function of the relaxation time is quite problematic, and, as far as we know, this formula does not exist for the moment. In order to analyze the relaxation process and make an unambiguous comparison with experiment possible, we introduce an exponential decay with a relaxation time parameter. Then we will look for a solution of the system

$$
\begin{aligned}
& \frac{\partial}{\partial x}\left(\chi \chi_{0} \frac{\partial \varphi(t)}{\partial x}\right)=-\rho(t), \\
& \rho(t)=-\frac{\partial P(t)}{\partial x}-\tau \frac{\partial \rho(t)}{\partial t},
\end{aligned}
$$

where $\varphi$ is a potential, $\rho$ is a density of the space charge, $\chi, \chi_{0}$ are the dielectric constants, and $\tau$ is a relaxation parameter. A magnitude of polarization $P$ is determined from Eq. (6). ${ }^{19}$

Let us consider first a fast relaxation process, which corresponds in the limit to $\tau=0$ and in this case the electric field $E$ and potential $\varphi$ can be derived analytically by solving a boundary problem. By doing so, one needs to integrate Eqs. (9) and (10) with zero potential at the boundary:

$$
\begin{gathered}
E=-\frac{\partial \varphi}{\partial x}=\frac{l_{0} P_{0}}{3 k_{B} \chi \chi_{0}}\left\{\frac{N}{T} \frac{\partial \varepsilon_{\mathrm{HO}}}{\partial x}-\left.\frac{1}{L} \frac{N \varepsilon_{\mathrm{HO}}}{T}\right|_{0} ^{L}\right\}, \\
\varphi=-\frac{l_{0} P_{0}}{3 k_{B} \chi \chi_{0}}\left\{\left.\frac{N \varepsilon_{\mathrm{HO}}}{T}\right|_{0} ^{x}-\left.\frac{x}{L} \frac{N \varepsilon_{\mathrm{HO}}}{T}\right|_{0} ^{L}\right\},
\end{gathered}
$$

where $\varepsilon_{\mathrm{HO}}=2 \varepsilon_{\mathrm{H}}-K \varepsilon_{\mathrm{O}}$ and $\left.\frac{1}{T} N \varepsilon_{\mathrm{HO}}\right|_{0} ^{L}=\left(\frac{1}{T} N \varepsilon_{\mathrm{HO}}\right)_{L}-\left(\frac{1}{T} N \varepsilon_{\mathrm{HO}}\right)_{0}$.

Equations (11) and (12) are the exact solutions if $N / T$ $=$ const (dipole concentration over temperature) does not depend upon the coordinate $x$. In fact, as will be shown below, $N / T$ depends on $x$ only slightly ( $N / T \approx$ const) and Eqs. (11) and (12) can be solved with an acceptable accuracy.

For dielectric materials, the polarization current is nothing but a bias current, hence it can be derived by differentiating electric field [Eq. (11)] over time using the relation $\partial / \partial t=$ $-\left(D-\nu_{1}\right) \partial / \partial \mathrm{x}$ :

$$
\begin{aligned}
j_{b}= & \chi \chi_{0} \frac{\partial E}{\partial t}=\frac{l_{0} P_{0}}{3 k_{B}}\left\{-\left(D-\nu_{1}\right) \frac{\partial}{\partial x}\left(\frac{N}{T} \frac{\partial \varepsilon_{\mathrm{HO}}}{\partial x}\right)-\left.\frac{\nu_{1}}{L^{2}} \frac{N \varepsilon_{\mathrm{HO}}}{T}\right|_{0} ^{L}\right. \\
& \left.+\left.\left(D-\nu_{1}\right) \frac{1}{L} \frac{\partial}{\partial x} \frac{N \varepsilon_{\mathrm{HO}}}{T}\right|_{0} ^{L}\right\} .
\end{aligned}
$$

It follows from the equation that the bias current is determined not only by total energy changes in the shock front [the first term in parentheses in Eq. (13)] but also by changes in energy at the boundaries of the sample [the last two terms in Eq. (13)]. More so, those two terms define the polarization current outside of the shock front, which is the current measured in the experiment.

Although the relaxation of the polarization charge in one form or another, as well as exponential polarization decay has been considered by Allison ${ }^{11}$ and later studied by many authors, ${ }^{20}$ satisfactory agreement with experimental measurements has not been obtained until now. We will show in the next section that it can be achieved if the density of the space polarization charge $\rho$ as a function of the relaxation time $(\tau \geqslant 0)$ is taken into account. As a result of this, the calculated current-time curve is in good qualitative and quantitative agreement with experiments by Eichelberger and Hauver. $^{1}$

\section{COMPARISON WITH EXPERIMENTS}

In the calculations, we use a model sample of length $L$ $=1 \mathrm{~mm}$. The amplitude of the shock wave was chosen as $10 \mathrm{GPa}$. Those parameters are presumably relevant to the experimental measurements by Eichelberger and Hauver in their pioneering work. ${ }^{1}$ The values of the impact wave velocity and mass velocity calculated by Eqs. (A8) and (A4) agree with experiment quite well for amplitudes lower than $1 \mathrm{GPa} .{ }^{21}$ Distributions of the polarization charge over the sample induced by the shock wave are derived from Eqs. (10) and (6) by using distribution functions from Eqs. (7) and (8). The potential distributions were obtained from the Poisson equation (9) with zero boundary conditions at the ends of the sample.

First, we analyze the fast relaxation processes with $\tau=0$ (see analytical solution in Sec. III). The calculated polarization charge and potential induced by the shock wave progressing across the sample are shown in Fig. 2. The distribution function for pressure in the shock wave as a function of time is shown in Fig. 2(a). Figure 2(b) depicts the process of space charge formation in the shock wave front that is propagating across the sample. This figure illustrates the fact that the space charge has positively and negatively charged regions that are well separated and that the positively charged region is ahead of the negatively charged region. This corresponds to the alignment of the water molecule with hydrogen atoms along with the wave movement. Potential formed by the polarization charge is zigzaglike as shown in Fig. 2(c), 

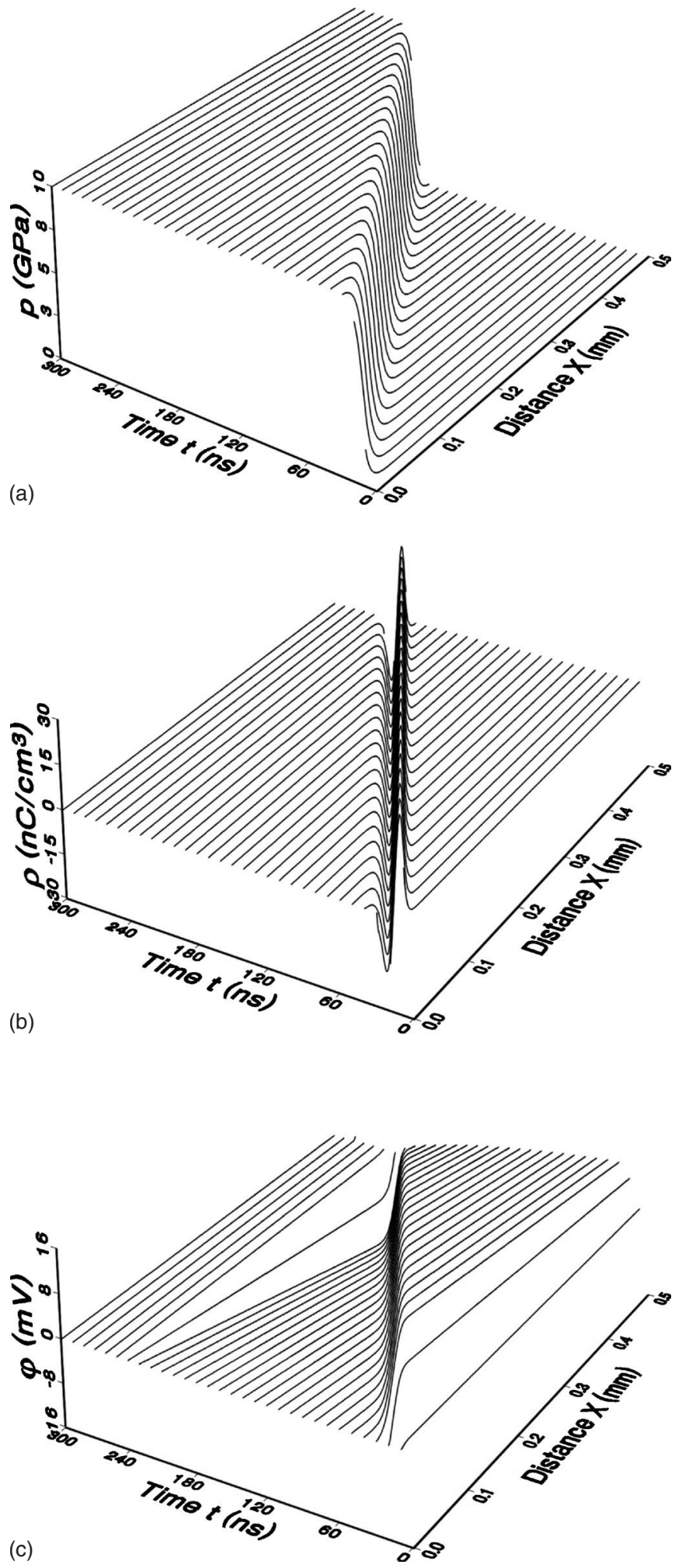

FIG. 2. Distribution functions of (a) shock-wave profile in the water sample, the amplitude of the shock wave is taken as $p_{1}$ $=10 \mathrm{GPa}$; (b) shock-induced polarization charge is shown assuming the fast charge relaxation process; (c) shock-induced potential formed by the polarization charge. The calculations were carried out in the Lagrange coordinate system coupled with mass velocity, i.e., using change of variable $x \rightarrow x-\left(D-\nu_{1}\right) t$ [see text, Sec. II, Eq. (11)].

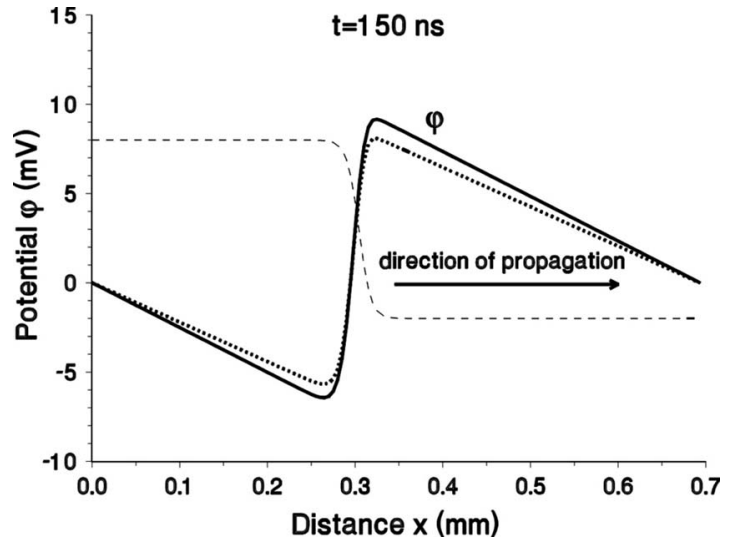

FIG. 3. The zigzag-shaped potential formed by the double charge is shown at $150 \mathrm{~ns}$. The results of numerical modeling (solid line) are compared with the analytical solutions (dotted lines). The shock wave profile with the amplitude of $10 \mathrm{GPa}$ is scaled to show in the same figure (dashed line).

with a pronounced maximum and minimum, which change their magnitudes with the shock wave progressing across the sample. Such a behavior of the shock wave, polarization charge, and potential is derived from both numerical simulation and analytical methods used here. For example, Fig. 3 shows a snapshot of the distribution function of the potential $\varphi$ at $150 \mathrm{~ns}$. It is clearly seen that numerical solutions obtained from Eqs. (9) and (10) (solid line) agree well with the analytical results of Eq. (12) (dotted line); a small deviation is due to variation of $N / T$.

The bias current calculated from Eq. (13) is shown in Fig. 4 in comparison with the current obtained from a numerical solution of Eqs. (9) and (10). From Fig. 4, the largest change in the bias current occurs at the very beginning of the polarization curve at the moment when the shock wave enters the sample, which is described by the last term in parentheses in Eq. (13). The shown peak corresponds to the formation of the double charge layer in the shock front. Once the double charge layer is formed and has started moving across the sample, the current is defined by the rate of the materials compression as described by the second term in parentheses

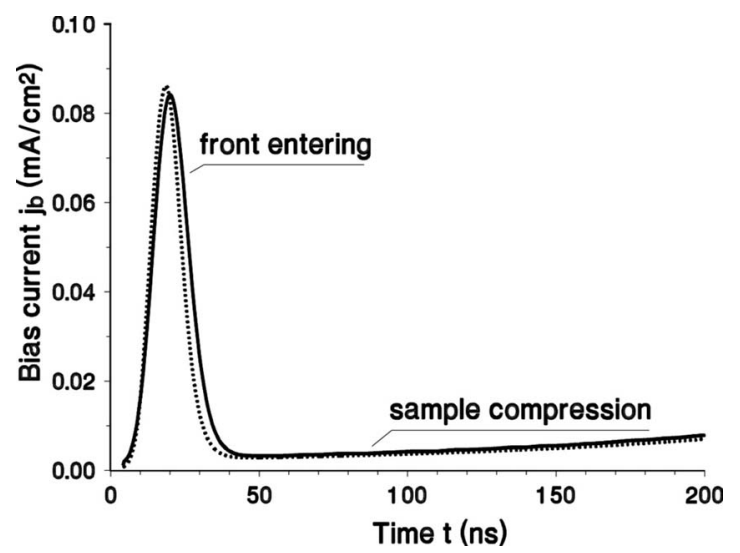

FIG. 4. The shock-induced bias current obtained from numerical modeling (solid line) is compared with the analytical solutions (dotted lines). 
Eq. (13). The small value of the bias current, which reaches only about $90 \mu \mathrm{A} / \mathrm{cm}^{2}$ even at its maximum, and the shape of the curve (at $\tau=0$ ) are very different from those observed in polarization measurements by Eichelberger and Hauver. ${ }^{1}$

We will show now that the results are in much better agreement with experiment once the slow relaxation process $(\tau>0)$ is considered. Figure 5 illustrates the results of the numerical simulation of space charge density, electric field, and potential at $150 \mathrm{~ns}$ for various values of the relaxation parameter $\tau$. The behavior of the potential function [Fig. 5(a)] and electric field [Fig. 5(b)] is significantly different from those described above because an increase of the relaxation time $\tau$ leads to an accumulation of the polarization charge [Fig. 5(c)] in the sample. This is reflected in, on one side, an increase in the positive charge maximum and, on the other side, in a leak of the negative charge through the grounded surface of the sample. The interaction of those two charges results in the appearance of a single charged state of the shock wave front and nonzero electric field distributed over the entire sample. Note that the double charged layer still exists at $\tau=10^{-8} \mathrm{~s}$ and $\tau=10^{-7} \mathrm{~s}$ but the charge of one sign only is observed at $\tau=10^{-6} \mathrm{~s}$. Such a slow relaxation, in fact, means that dipole molecules remain aligned for some time after the wave front has left the sample. The zigzagshaped potential is transformed into a corner shaped function with one pronounced maximum at $\tau=10^{-6} \mathrm{~s}$ and two linear pieces.

The calculated current is quite large and reaches $10 \mathrm{~mA} / \mathrm{cm}^{2}$ (in comparison with the case of the fast relaxation process $\tau=0$, where the current is $90 \mu \mathrm{A} / \mathrm{cm}^{2}$ ) and is in good agreement with experiment. ${ }^{1}$ In calculations we used a voltage of $0.5 \mathrm{~V}$, external resistance was taken as $10 \mathrm{Ohm}$ and surface area of the sample was taken as $5 \mathrm{~cm}^{2}$ (those are estimated as reasonable parameters of the experiments). ${ }^{1}$ The current-time feature is also affected by the process of charge accumulation: the slower the relaxation time, the more charge is accumulated in the sample (Fig. 6). Thus, only one maximum is observed for $\tau=10^{-8} \mathrm{~s}$ that corresponds to the double charged layer formed [see Fig. 5(c)], which is largely similar to the fast relaxation results of $\tau=0 \mathrm{~s}$ (also see Fig. 4). Once the system is allowed to relax for a sufficient amount of time $\left(\tau \geqslant 10^{-6} \mathrm{~s}\right)$, it exhibits significantly different behavior of the current, which first grows rapidly and then continues to grow at a slower rate (Fig. 6). This trend is in remarkable agreement with the experiment. ${ }^{1}$ Furthermore, visually this current-time curve is also similar to the currenttime signal resulting from shock orientation in plastic. ${ }^{1}$ The similarity of the shape of current-time signals in both water and plastic may suggest that the orientation polarization has a dominating contribution into polarization signal in other materials constituting from polar molecules due to shockinduced orientation of dipoles. For comparison, Fig. 6 demonstrates the bias current curves obtained from the model by Allison for the selected relaxation times. Both the model developed here and the model by Allison yield similar currents in a stationary region when the shock wave is progressing inside the sample. Unlike the model presented here, Allison's method does not describe a rapid current increase when the wave enters the sample. On the other hand, the only difference between the calculated and experimental
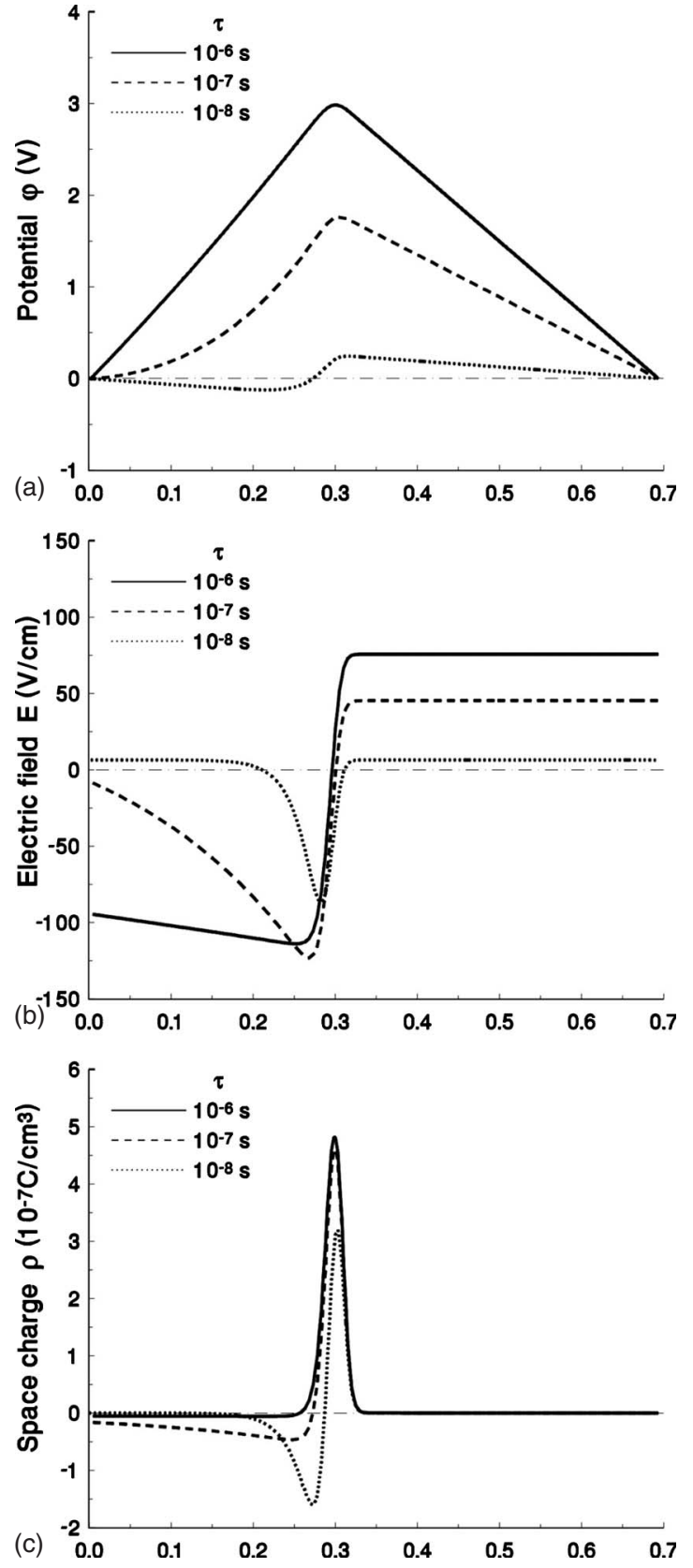

FIG. 5. Distribution function of (a) shock-induced potential, (b) shock-induced electric field, (c) shock-induced space charge in a water sample at $150 \mathrm{~ns}$ for different values of relaxation parameter $\tau$. The slow relaxation of polarization charge causes the charge buildup and leads to the formation of a single charged layer within the shock wave front (shown by the solid line) instead of the double charged layer (shown by the dotted line), which is characteristic for fast relaxation processes. This induces the electric field in a sample and transforms the zigzaglike potential (dotted line) into a piecewise linear function with a pronounced maximum (solid line).

curves is a sharp peak at the beginning of the current characteristics, which perhaps implies the existence of an additional polarization mechanism related most likely to a fast relaxation process since the process does not give any significant contribution to current at longer times. Noting an increase in conductivity at $P \geqslant 10 \mathrm{GPa},{ }^{22,23}$ one can specu- 


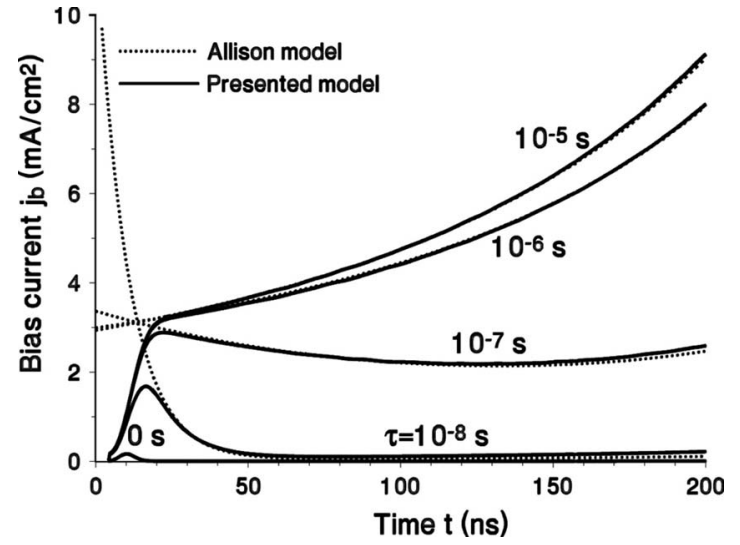

FIG. 6. The bias current created by the polarization charge. The continuous increase of the calculated current at large charge relaxation times points out to a buildup process of the polarization charge in the sample.

late that the peak corresponds to an appearance of electrons, protons, and/or other quickly relaxing charges in the system. Although this contribution is not described by our analytic model, we made an attempt to mimic the experimental polarization results based on this proposition. Hence, the polarization current can be reproduced if one assumes that the net signal is complex and is formed by a combination of both quickly and slowly relaxing polarization components as illustrated in Fig. 7. The significant increase of the polarization current at the later stages of the polarization signal (Fig. 7) is defined by the increase of the dielectric permittivity ${ }^{24}$ and/or the electric conductivity ${ }^{22,23}$ behind the wave front.

Figure 7 also shows that the rise time (which is the time of the initial growth of the polarization signal) for water is $30 \mathrm{~ns}$, which agrees well with experiment. This duration of

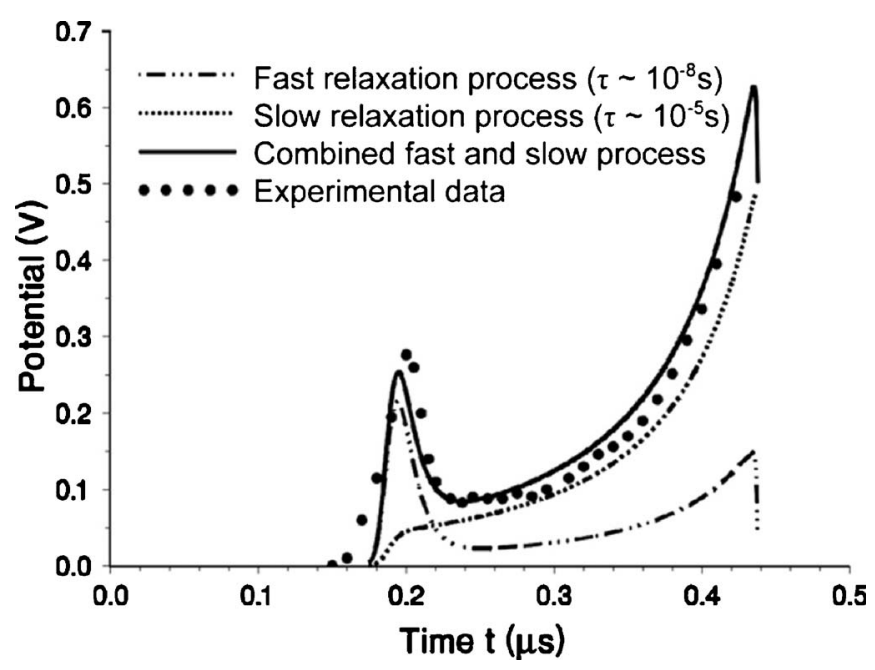

FIG. 7. The electrostatic potential as a function of time is shown for the experimental data by Eichelberger and Hauver (Ref. 1) and calculated results. It is shown that a combined result of a slowly relaxing polarization process (the dipole polarization) and a quickly relaxing polarization process (for example, the diffusion of free charge carriers in the wave front) in water yields good agreement with experiment.

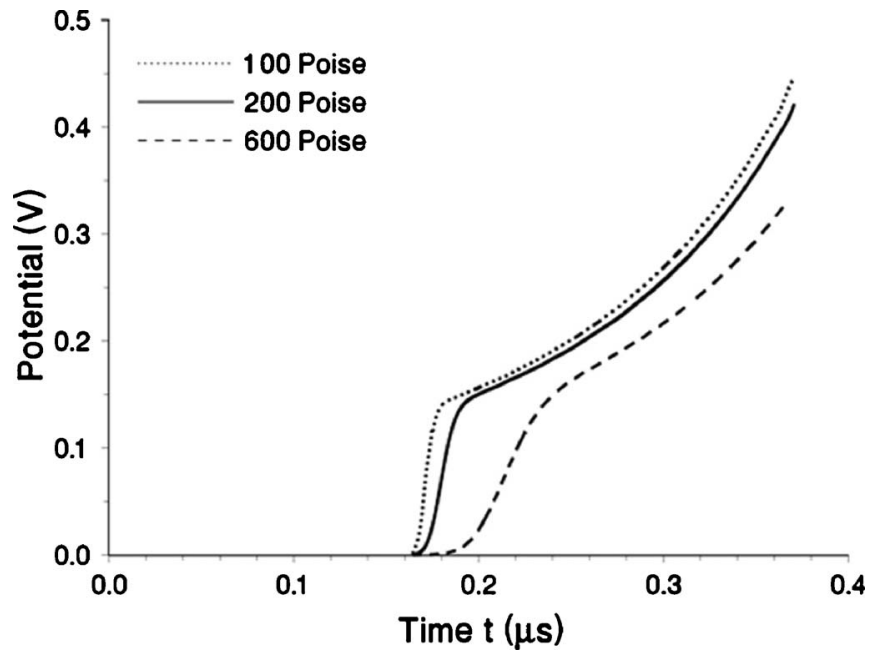

FIG. 8. Voltage-time feature is shown to compare directly to the experiments by Eichelberger and Hauver. External resistance is taken as $10 \mathrm{Ohm}$, surface area of the water sample is $5 \mathrm{~cm}^{2}$; viscosity of water is estimated as 200 Poise.

time is necessary to form a wave front $28 \mu \mathrm{m}$ wide and also to create an initial polarization charge, which indicates that the polarization charge is largely formed by the wide $(\mu \mathrm{m})$ part of the wave front (also see the Appendix) and not by the narrow $(\mathrm{nm})$ part as has been thought earlier. ${ }^{20}$ Should one assume that the polarization charge is to be formed by a nanometer layer of the wave front, the time of the polarization signal growth has to be much shorter, on the order of a few picoseconds, which is inconsistent with the experiment.

An analysis of the shock front width can also provide a wealth of information regarding the viscosity of materials exposed to shock excitation. Thus, the front width of $28 \mu \mathrm{m}$ obtained here corresponds to the maximum viscosity of water 200 Poise, which is slightly below $10^{4}$ Poise obtained by Sakharov's method ${ }^{25}$ of the "corrugated front" and slightly above 20 Poise reported by Altshuler, ${ }^{26}$ where a more direct method involving the accelerated cylinders was used. Note that the value of 20 Poise is given for pressure of $6.6 \mathrm{GPa}$, whereas at the pressure above $10 \mathrm{GPa}$ water acquires a polymeric structure, ${ }^{26}$ which necessarily causes a jump in viscosity.

An estimate of Debye relaxation time ${ }^{17}$ as $\tau=4 \pi \xi a^{3} / k_{B} T$ (where $a$ is a molecular radius at 200 Poise viscosity $\xi$ ) yields $\tau \sim 10^{-5} \mathrm{~s}(a=1.4 \AA, T=896 \mathrm{~K}$ at $10 \mathrm{GPa})$, which is consistent with the description of the slowly relaxing process (Fig. 7). A more precise comparison with experiment is currently problematic and ambiguous since data on viscosity and, more importantly, direct measurements of the width of the impact wave front in water are lacking. However, a sensitivity of the polarization signal to a change of the front width can be demonstrated as shown in Fig. 8, where dynamics of the current-time characteristics of a slowly relaxing polarization process is studied as a function of the wave front width. From Fig. 8, the front width, indeed, determines the most rapid initial region of the current climb and, hence, the front width can be established based on the current rise-time data. An influence of the front width is not so strongly pro- 
nounced in the stationary region, where the Allison model is valid. Although a decline in the current takes place, this is mostly related to a decrease of gradients of the velocity and electric field within the wave front, and consequently forces exerted on the molecules. Taking into account both the fact that a material's viscosity changes by orders of magnitude behind the shock front and also quite a precise prediction of water viscosity obtained here, one can suggest that a potentially accurate novel method for determining a material's viscosity can be developed based on the analysis of polarization signals in shocked samples.

\section{DISCUSSION}

In this article we presented a consistent mechanism to explain shock-induced polarization in distilled water. It is assumed in this mechanism that the shock wave-induced stress and inertial field orients dipole water molecules in such a way that the $\mathrm{H}$ atoms are always moving along with the shock wave in front of $\mathrm{O}$ atoms. In order to analyze this effect quantitatively, a method of calculating the shock wave structure has been developed (see the Appendix). Expressions were derived to couple the gradients of the stress and kinetic components of the total energy with the stress and inertial forces exerted on individual atoms in the water molecule. Further, it was noticed that the orientation polarization of water in the shock wave can be described by the Langevin equation similarly to the description of the dipole polarization in electric fields. Based on this notion, the method for calculating the polarization has been developed (Sec. II). In order to directly compare the results with experiment and suggest an interpretation of the measured polarization, the method for calculating the potential of the electric field and bias current has been worked out by using the polarization charge density derived from the calculated polarization (Sec. III). It has been established that the space polarization charge decay behind the shock front plays an important role in the polarization phenomena; therefore, a relaxation time parameter $\tau$, which is a function of the water viscosity, has been introduced. The polarization currents calculated for large values of $\tau$ exhibit good qualitative and quantitative agreement with measured polarization signals by Eichelberger and Hauver. We propose that the large values of currents obtained are closely related to large values of water viscosity behind the shock front.

The viscosity, most likely, is a major factor in determining the relaxation parameter $\tau$, which in turn defines the shape and value of the polarization charge. When the viscosity is small, which also means that $\tau$ is small, the charge behind the shock front relaxes rapidly and the current falls fairly quickly after the initial rise, forming a maximum at the very beginning of the polarization curve. The polarization charge does not have enough time to accumulate and localizes only within the shock front. The charged front while moving across the sample does not contribute to the polarization signal.

Once the viscosity of the compressing material reaches large values, the charge relaxation time also becomes large. This, in turn, causes the polarization current to continue growing while the shock front progresses across the sample, i.e., the polarization charge accumulates within the entire sample. We believe that such a process takes place in the experiments by Eichelberger and Hauver since the polarization current calculated in our model exhibits the same features as in their experimental work: (1) the initial very rapid rise in current, (2) a further, more gradual rise in current, with an increasing rate of change, and (3) an essentially instant drop of current when the shock wave leaves the sample.

The theory of dipole polarization developed here, however, does not replicate all details of the experimental plot. An initial peak of polarization current registered in the experiment does not appear in our calculations. As follows from our theory, this peak cannot be described by slow relaxation processes (large $\tau$ ) within our method. From this an important conclusion follows; the experimental polarization curve cannot be described by the orientation polarization alone, although this orientation mechanism of polarization defines the main features of the current-time curve. One can suggest that there is another mechanism contributing to the polarization signal at the beginning of the current-time curve, in fact, similarly to the fast relaxation processes considered in the proposed model (see Fig. 4). Alternatively, there may be a process that gives a contribution to current measurements but is not related to the dipole polarization, for example, a diffusive redistribution of free charges due to the shock wave.

An effect of dissociation of water molecules under equilibrium and elevated pressures is neglected in this work. It is difficult to perform accurate modeling of this sort at this time, but one can speculate in simple terms that in order to make an impact on shock induced polarization, the concentration of dissociated water molecules should be very high, on the order of $10^{19}-10^{20}$ per cm. ${ }^{3}$ Alternatively, the diffusion coefficients of $\mathrm{H}^{+}$and $\mathrm{OH}^{-}$ions should be anomalously high.

It is worthwhile to comment on the limitations and perspectives of the theory developed here. In this work, a relatively simple analytical method for the calculation and analysis of the structure of a stationary impact wave from the induced polarization is developed. The method accurately describes a stationary wave when the wave front is inside a sample at constant pressure at the edge. Undoubtedly, it is interesting, although more challenging, to simulate nonstationary waves; for example, a process of the formation of the wave front while entering and leaving the sample, or to reflect dynamics of the pressure at the edge of the sample. Modeling such processes requires a numerical solution of the conservation laws of mass, impulse, and energy; rewardingly, it will open a possibility to analyze with a great deal of accuracy the polarization phenomena in detonation waves.

\section{CONCLUSION}

A consistent method for calculation of the orientation polarization in shocked water is developed. The structure of the shock wave front is described analytically and used for analysis of polarization. The origin of the water polarization is in the interplay between stress and inertial forces exerted 
on each individual atom in the sample; therefore, net polarization is calculated via the stress and inertial contributions to the total energy of atoms and parameters of the shock wave. Further, the polarization charge is obtained from the derived polarization; and the density of the polarization charge is used to determine other polarization characteristics such as potential, electric field, and the bias current. The results obtained for the shock-induced current agree well with experimental measurements by Eichelberger and Hauver. The correspondence observed leads us to suggest that the dipole polarization is a dominating polarization mechanism in shocked water. The similarity of the shape of the currenttime characteristics and experimentally observed currents for plastics indicates that the methodology proposed here can be generalized to other materials built out of dipole molecules.

\section{ACKNOWLEDGMENTS}

This work was supported in part by ARO MURI (Grant No. W9011NF-05-1-0266). M.K. is grateful to the DMR of the National Science Foundation for support under the Independent Research and Development Program. Any appearance of findings and conclusions, expressed in this material are those of the authors and do not necessarily reflect views of the NSF.

\section{APPENDIX: STRUCTURE OF THE SHOCK WAVE FRONT IN WATER}

An analysis of the shock wave front structure provides very important information. In particular, distribution functions of mass, velocity, and pressure over the shock front make it possible to calculate the kinetic and potential energy per molecule and/or, which is more important, per fragment of a molecule. Those characteristics are crucial in determining a polarization charge induced by the shock front in a material constituted of polar molecules. The shock front width is also an important feature since, as is established below, it controls the shape of the polarization signal at the entry of the shock front in a sample.

Let us assume that the equation of state (EOS) of a condensed matter is given by the expression ${ }^{27}$

$$
p=\rho_{0} C^{2} \mu f(\mu)+\gamma_{0} \rho_{0} e,
$$

where $\mu=1-\rho_{0} / \rho ; \rho, \rho_{0}$ are the current and initial densities, $p$ is the pressure, $e$ is the internal energy, $C$ is the linear speed of sound at ambient conditions, and a function $f(\mu)$ depends upon $\gamma_{0}$ and $S$, the Grüneisen coefficients. Then, the structure of the shock wave front in a viscous liquid is determined from the conservation laws of mass, impulse, and energy similarly to the gas-phase state $\mathrm{e}^{28-30}$

$$
\begin{gathered}
p=p_{0}+\rho_{0} D v+\sigma_{v}, \\
\sigma_{v}=-\frac{1}{2} \gamma_{0} \rho_{0} D^{2} \mu\left(\mu_{1}-\mu\right), \\
\nu=D \mu,
\end{gathered}
$$

where $\sigma_{v}$ is the viscous stress; $D$ and $v$ are the shock and mass velocities. The density distribution within the shock front is described by a first order ordinary differential equation

$$
\frac{\partial \mu}{\partial x}=-\frac{\gamma_{0} D \rho_{0}}{4 \xi} \mu\left(\mu_{1}-\mu\right) W(\mu),
$$

with

$$
W(\mu)=\frac{2}{\gamma_{0}\left(\mu_{1}-\mu\right)}\left\{1-\frac{U^{2}(\mu)}{D^{2}\left(\mu_{1}\right)}\right\}-\frac{\mu}{\mu_{1}-\mu} .
$$

Here $\mu_{1}=1-\rho_{0} / \rho_{1}, \rho_{1}$ is the density maximum of the compressed material behind the shock wave front and and $\xi$ is the viscosity. The speed of sound in compressed matter $U$ and the speed of the shock wave $D$ are expressed as follows:

$$
\begin{gathered}
U^{2}(\mu)=\frac{\gamma_{0} p_{0}}{\rho_{0}}+C^{2} f(\mu), \\
D^{2}\left(\mu_{1}\right)=\frac{\gamma_{0}}{2 \rho_{0}}\left(p_{1}+p_{0}\right)+C^{2} f\left(\mu_{1}\right) .
\end{gathered}
$$

An equation for $\mu_{1}$ can be defined from the condition $\partial \mu / \partial x=0$ at the point $\mu=\mu_{1}$ [see Eqs. (A5) and (A6)]. Then, the solution of the system (A7) and (A8) yields the shock Hugoniot in the form

$$
\rho C^{2} \mu_{1} f\left(\mu_{1}\right)=p_{1}\left(1-\frac{\gamma_{0}}{2} \mu_{1}\right)-p_{0}\left(1+\frac{\gamma_{0}}{2} \mu_{1}\right) .
$$

Once Eq. (A9) is solved for $\mu_{1}$, one can find the density distribution over the wave front and other parameters from Eqs. (A2) and (A4) by solving Eq. (A5) numerically. Alternatively, Eq. (A5) can be integrated analytically if $W$ remains constant. By choosing $W$ in the middle of the wave front, one gets

$$
\mu=\frac{\mu_{1}}{1+\exp \left(\frac{4 x}{H}\right)},
$$

where

$$
H=\frac{16 \xi}{\gamma_{0} D \rho_{0} \mu_{1} W\left(0.5 \mu_{1}\right)}
$$

is the front width. Equations (A10) and (A11) accompanied by Eqs. (A1)-(A4) entirely define the parameters of the shock wave and its structure. Should the $f(\mu)$ function of $\mathrm{H}_{2} \mathrm{O}$ be chosen in the form ${ }^{27} f(\mu)=\left(1-0.5 \gamma_{0} \mu\right) /(1-S \mu)$, and assuming $p_{0}=0$ when $p_{1} \gg p_{0}$, then $\mu_{1}, D$, and $\nu_{1}$ can be consequently determined from Eqs. (A9), (A8), and (A4), respectively, as $\mu_{1}=p_{1 n} /\left(1+S p_{1 n}\right), D=C\left(1+S p_{1 n}\right)^{1 / 2}$ and $v_{1}$ $=p_{1 n} C /\left(1+S p_{1 n}\right)^{1 / 2}$, where $p_{1 n}=p_{1} / \rho_{0} C^{2}$. Here, water parameters $\rho_{0}=1.0 \mathrm{~g} / \mathrm{cm}^{3}, \quad \gamma_{0}=1.64, \quad \mathrm{~S}=1.786, \quad$ and $\mathrm{C}$ $=1.489 \mathrm{~km} / \mathrm{s}$ were taken from Ref. 27

Note, the derived analytical formulas are valid only in a narrow range of pressures; for example, from 1 to $10 \mathrm{GPa}$, in which viscosity can be considered as a maximal and approximately constant value. Actually, the range of pressures associated with the shock wave propagation in the material is much wider, from ambient pressure $\left(10^{5} \mathrm{~Pa}\right)$ to $1 \mathrm{GPa}$ be- 
cause the material's viscosity changes by several orders of magnitude and, therefore, the pressure gradient is very large. As is well known, the structure of the shock wave front in water can be represented as a region (usually a few microns wide) of rapidly growing pressure (pressure ranges from $10^{5} \mathrm{~Pa}$ to $1 \mathrm{GPa}$ ) and then a region (a few nanometers wide) of slowly growing pressure (pressure ranges from 1 to $10 \mathrm{GPa}) .{ }^{31,32}$ The existence of those two regions of the shock front is determined by the nonmonotonic dependence of viscosity on pressure and is characteristic for condensed matter. From the knowledge of this dependence, the entire shock front (including both regions) can be defined by solving Eq. (A5). We are much more interested here in analyzing the gradually increasing viscosity region, which we will call hereafter a viscous region, because this part of the shock front largely contributes to the shock-induced polarization.
*Author to whom correspondence should be addressed.

${ }^{1}$ R. J. Eichelberger and G. E. Hauver, in Les Ondes de Detonation (Publicadu Centre National de la Research Scientifique, Paris, 1962), p. 361.

${ }^{2}$ P. Harris, J. Appl. Phys. 36, 739 (1965).

${ }^{3}$ Y. Horie, Br. J. Appl. Phys. 1, 1183 (1968).

${ }^{4}$ P. Harris and H. N. Presles, J. Chem. Phys. 77, 5157 (1982).

${ }^{5}$ K. Saxena, Y. Hironaka, H. Hirai, and K. Kondo, Appl. Phys. Lett. 68, 920 (1996).

${ }^{6}$ T. Ogura, Y. Hironaka, F. M. Nicol, K. G. Nakamura, and K. Kondo, Jpn. J. Appl. Phys., Part 1 40, 2378 (2001).

${ }^{7}$ A. G. Antipenko, A. N. Dremin, S. S. Nabatov, and V. V. Yakushev, Fiz. Goreniya Vzryva 11, 438 (1975).

${ }^{8}$ A. I. Goncharov and S. P. Soloviev, Combust., Explos. Shock Waves 40, 658 (2004).

${ }^{9}$ A. G. Ivanov, E. Z. Novitski, V. N. Mineev, Yu. V. Lisitsyn, Yu. N. Tyunyaev, and G. I. Bezrukov, Sov. Phys. JETP 53, 41 (1967).

${ }^{10}$ V. N. Mineev, A. G. Ivanov, Yu. V. Lisitsyn, E. Z. Novitski, and Yu. N. Tyunyaev, Sov. Phys. JETP 59, 1091 (1970).

${ }^{11}$ F. E. Allison, J. Appl. Phys. 36, 2111 (1965).

${ }^{12}$ Ya. B. Zel'dovich, Sov. Phys. JETP 53, 237 (1967).

${ }^{13}$ R. M. Zeydel, Sov. Phys. JETP 54, 1253 (1968).

${ }^{14}$ A. G. Antipenko, S. S. Nabatov, and V. V. Yakushev, Fiz. Goreniya Vzryva 11, 3 (1975).

${ }^{15}$ Yu. Skryl and M. M. Kuklja, Phys. Rev. B 71, 094109 (2005).

${ }^{16}$ Yuri Skryl, Anna A. Belak, and Maija M. Kuklja, Shock Induced Polarization in Binary Electrolytes, AIP Conf. Proc. No. 845, edited by M. D. Furnish, M. Elert, T. P. Russell, and C. T. White (AIP, Melville, 2006), pp. 355-358.

${ }^{17}$ Charles Kittel, Introduction to Solid State Physics, 7th ed. (Wiley, New York, 1996).

${ }^{18}$ E. U. Enikeev, S. I. Kubarev, and O. A. Ponamarev, Combust., Explos. Shock Waves 23, 440 (1987).
${ }^{19}$ Note that the differential form Eq. (10) for the relaxation charge is valid only for small magnitudes of $\tau$, for large $\tau$, we used numerical methods.

${ }^{20}$ V. N. Mineev and A. G. Ivanov, Usp. Fiz. Nauk 119, 75 (1976).

${ }^{21}$ K. Nagayama, Y. Mori, K. Shimada, and M Nakahara, in Shock Compression of Condensed Matter, edited by Furnish, Chabildas, and Hixson (American Institute of Physics, Melville, 2000), p. 65 .

${ }^{22}$ S. D. Hamann and M. Linton, Trans. Faraday Soc. 62, 2234 (1966); 65, 2186 (1969).

${ }^{23}$ W. B. Holzapfel, J. Chem. Phys. 50, 4424 (1969).

${ }^{24}$ For example, an excellent fit to experimental data can be obtained if dielectric permittivity of water behind the front is nearly doubled, $\chi=154$ as compared to $\chi=78.54$ under normal conditions.

${ }^{25}$ A. D. Sakharov, R. M. Zeydel, V. N. Mineev, and A. G. Olejnik, Dokl. Akad. Nauk SSSR 159, 1019 (1957).

${ }^{26}$ L. V. Altshuler, in Shock Compression of Condensed Matter1991, edited by Schmidt, Dick, Forbes, and Tasker (Elsevier, New York, 1992), P. 509.

${ }^{27}$ S. Itoh, Y. Natamitsy, Z. Y. Liu, and M. Fujita in Shock Compression of Condensed Matter, edited by Schmidt, Dandekar, and Forbes (1998), p. 195.

${ }^{28}$ A. A. Samarsky and Yu. P. Popov, Difference Schemes in Gas Dynamics (Nauka, Moscow, 1985) (in Russian).

${ }^{29}$ G. B. Whitham, Linear and Nonlinear Waves (Wiley, New York, 1976).

${ }^{30}$ R. Courant and K. O. Friedrichs, Supersonic Flow and Shock Waves (Springer, New York, 1999).

${ }^{31}$ V. N. Mineev and R. M. Zeydel, Sov. Phys. JETP 54, 1633 (1968).

${ }^{32}$ Ya. B. Zel'dovich, S. B. Kormer, M. B. Sinicyn, and K. B. Yushko, Dokl. Akad. Nauk SSSR 138, 1333 (1961). 